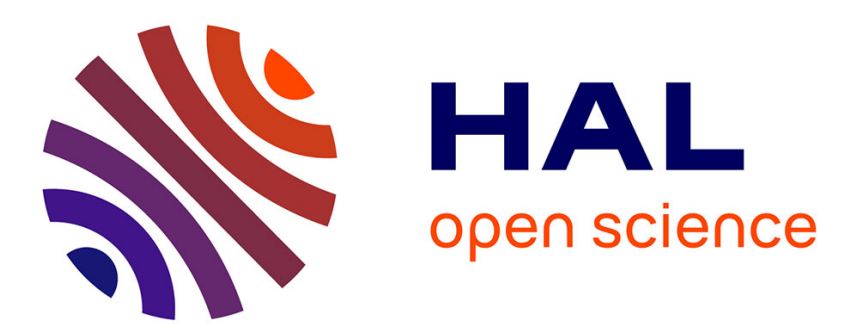

\title{
Towards a Better Understanding of Photo-excited Spin Alignment Processes Using Silole Diradicals.
}

Nans Roques, Philippe Gerbier, Yoshio Teki, Sylvie Choua, Petra Lesniakova, Jean-Pascal Sutter, Philippe Guionneau, Christian Guérin

\section{- To cite this version:}

Nans Roques, Philippe Gerbier, Yoshio Teki, Sylvie Choua, Petra Lesniakova, et al.. Towards a Better Understanding of Photo-excited Spin Alignment Processes Using Silole Diradicals.. New Journal of Chemistry, 2006, 30 (9), pp.1319-1326. 10.1039/b606593g · hal-00186603

\section{HAL Id: hal-00186603 https://hal.science/hal-00186603}

Submitted on 12 Nov 2007

HAL is a multi-disciplinary open access archive for the deposit and dissemination of scientific research documents, whether they are published or not. The documents may come from teaching and research institutions in France or abroad, or from public or private research centers.
L'archive ouverte pluridisciplinaire HAL, est destinée au dépôt et à la diffusion de documents scientifiques de niveau recherche, publiés ou non, émanant des établissements d'enseignement et de recherche français ou étrangers, des laboratoires publics ou privés. 


\title{
Towards a Better Understanding of Photo-excited Spin Alignment Processes Using Silole Diradicals
}

\author{
Nans Roques, ${ }^{a}$ Philippe Gerbier, ${ }^{a}{ }^{a}$ Yoshio Teki, ${ }^{* b}$ Sylvie Choua, ${ }^{c}$ Petra Lesniakovà, ${ }^{c}$ Jean-Pascal Sutter, ${ }^{d}$ \\ Philippe Guionneau, ${ }^{e}$ and Christian Guérin. ${ }^{a}$
}

\author{
Receipt/Acceptance Data [DO NOT ALTER/DELETE THIS TEXT] \\ Publication data [DO NOT ALTER/DELETE THIS TEXT] \\ DOI: 10.1039/b000000x [DO NOT ALTER/DELETE THIS TEXT]
}

The synthesis of two nitroxide-based diradicals connected to a 2,3,4,5-tetraphenylsilole (TPS) unit, especially designed to present high spin photo-excited states, is reported. While the bisnitronylnitroxide (NN) silole-based diradical, TPSNN, is unstable and 10 experiences a spontaneous fragmentation of its imidazolinic ring into a iso-butylammomium salt, the corresponding bisiminonitroxide (IN), TPSIN, is stable both in solution and in the solid state. This diradical crystallizes in the triclinic $P_{-1}$ space group with $a=10.984(1), b=11.474(1), c=17.492(1) \AA, \alpha=81.10(1), \beta=89.01(1)$, and $\gamma=65.71(2)^{\circ}$. Ground state magnetic properties of TPSIN have been investigated by means of SQUID and ESR measurements: the diradical displays weak intramolecular antiferromagnetic interactions $\left(J / k_{B} \approx-1 \mathrm{~K}\right)$, in agreement with its topology and with the molecular packing 15 observed in its crystal structure. In order to investigate the magnetic photo-excited states of TPSIN, time-resolved ESR experiments (TRESR) have been performed on this radical species. Despite the presence of both an appropriate topology for the diradical and a triplet photo-excited state for the TPS coupler, no TRESR signal was observed for this molecule within the timescale of the measurement. In addition to the work already published in this field, this result clearly indicates that besides the radical nature, the $\pi$-topological requirements and the need of photo-tunable spin-states for the coupler, the flexibility of the 20 molecule also plays a crucial role in the achievement of photo-induced spin alignment processes.

\section{Introduction}

Control of intramolecular spin alignment and exchange interactions in purely organic spin systems are essential topics 25 in the field of molecule-based magnetism. ${ }^{1,2}$ Since most studies are limited to the magnetic ground state, synthetic efforts are currently devoted to the design of high-spin compounds, in which an appropriate topology gives rise to ferromagnetic interactions between the spin bearing units. ${ }^{3-5}$ 30 Therefore, topologies expected to allow antiferromagnetic interactions, yielding a singlet or low spin ground state, have been generally discredited. The recent observation, for purely organic $\pi$-conjugated diradicals, of photo-excited quintet $(S=2)$ states starting from singlet ground state has relaunched 35 the interest in designing systems with the "wrong" topology. ${ }^{6-}$ ${ }^{13}$ Such systems are generated through photo-induced spin alignment using the $\pi$-conjugation between dangling iminonitroxide or verdazyl radicals $(S=1 / 2)$ and the photoexcited triplet $(S=1)$ state of 9,10-diphenylanthracene (Figure 40 1). Excited high-spin systems arising from the radical-triplet pairs have also been reported in the pioneering works of

\footnotetext{
${ }^{a}$ Laboratoire de Chimie Moléculaire et Organisation du Solide-UMRCNRS / UM2 5637, Université Montpellier II, C.C.007, Place E. Bataillon, 34095 Montpellier Cedex 5, France.Fax : +33.4.67.14.3852 ; Tel :+33.4.67.143.972;E-mail: gerbier@univ-montp2.fr

${ }^{b}$ Department of Material Science, Graduate School of Science, Osaka City University, 3-3-138 Sugimoto Sumiyoshi-ku, Osaka 558-8585, Japan. Fax:+81-6605-8585;E-mai: teki@sci.osaka-cu.ac.jp

${ }^{c}$ Institut Charles Sadron-UPR-CNRS 22,Université Louis Pasteur, 6 Rue Boussingault - BP 40016 - 67083 Strasbourg Cedex, France. Fax +33.3.88.414.099; Tel : +33.3.88.414.000 ; E-mail: turek@ics.ustrasbg.fr

${ }^{d}$ Laboratoire de Chimie de Coordination-UPR-CNRS 8241, Université Paul Sabatier, 205 route de Narbonne, 31077 Toulouse Cedex 4, France. E-mail: sutter@lcc-toulouse.fr

e Institut de Chimie de la Matière Condensée - UPR-CNRS 9048,

Université Bordeaux I, 87, Av. Dr. Schweitzer, 33608 Pessac, France. Tel: +33.5.40.002.279; E-mail:guio@icmcb-bordeaux.cnrs.fr
}

Corvaja et al. ${ }^{14}$ and Yamauchi et al. ${ }^{15}$ However, the number of references found in the literature is fairly limited, and in almost all the studies, stable nitroxide radicals have been used 45 as spin bearing units. For purely organic excited high-spin systems, only fullerene, anthracene and pyrene derivatives have been reported as couplers. The search for novel photoexcited high-spin organic systems constructed from both different radicals and triplet couplers is therefore an important 50 research target.

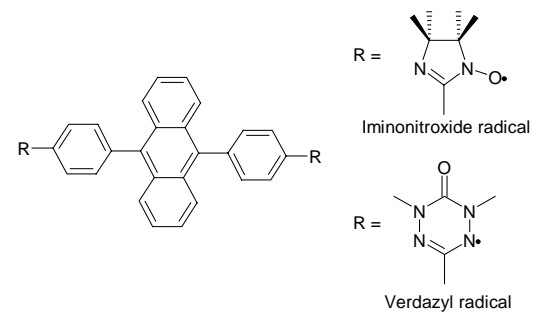

Fig. 1 Organic spin systems derived from 9,10-diphenylanthracene in which the photo-excited spin alignment has been observed.

In this respect, we decided to investigate the potentiality of the silole to act as a photo-active magnetic coupler. With this idea, we first studied and evidenced the presence of an 55 accessible photo-excited triplet state for the 2,3,4,5tetraphenyl-1,1-dimethylsilole (TPS). ${ }^{16}$ We also reported previously the synthesis, crystal structure, and ground state magnetic properties of a series of siloles bearing two nitroxide radicals, ${ }^{17,18}$ as well as the magnetic behaviour upon light 60 irradiation of one of them, the bis tert-butylnitroxide derivative (TPSNO). ${ }^{16}$ Briefly, the ground state magnetic behaviour of TPSNO is characterized by weak intramolecular antiferromagnetic interactions leading to a singlet ground state. Surprisingly, and despite the existence of a photo${ }_{65}$ excited triplet state for the parent TPS, no higher spin state was observed for the TPSNO diradical. Assuming that tert- 
butylnitroxide groups were not adequate for this purpose, we decided to graft nitronylnitroxide and iminonitroxide spinbearing units on the silole ring. The choice of these groups 70 was motivated by the fact that they have already allowed the successful spin alignment in the photo-excited states of $\pi$ conjugated diradical systems derived from the 1,9diphenylanthracene pattern. ${ }^{7-13}$ The design, synthesis, structural and electronic characterizations of a new silole-

75 linked iminonitroxide diradical is presented here in detail. Its magnetic behaviour has been investigated both in the ground and photo-excited states to explore the ability of the silole to act as a photo-active magnetic coupler.
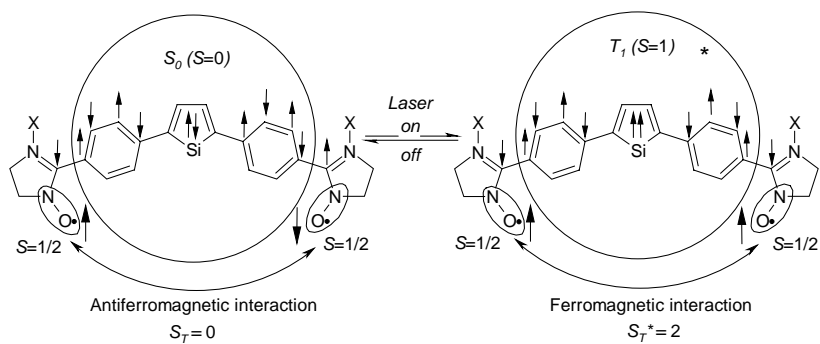

Fig. 2 Expected photo-induced spin alignment and sign inversion of the effective exchange between two dangling radical spins. TPSNN: $\mathrm{X}$ is an oxygen atom, and TPSIN: $\mathrm{X}$ is a lone pair. The phenyl rings present at the 3- and 4-positions and the methyl groups borne by the silicon atom have been removed by sake of clarity.

\section{Results}

\section{${ }_{80}$ Molecular design}

$\pi$-conjugated spin systems constructed from aromatic hydrocarbons and dangling stable radicals are ideal models to study the relationship between $\pi$ topology and spin alignment in photo-excited states. The design of the delocalized $\pi$-orbital 85 network is of the utmost importance in order to try to anticipate the spin state for both the ground state and the photo-excited state of the molecule. ${ }^{19}$ In photo-excited highspin states the nature of the magnetic exchange coupling between two dangling radical spins through the spin coupler ${ }_{90}$ changes from antiferromagnetic to ferromagnetic after photoexcitation. The key process, which is an enhanced intersystem crossing (ISC) mechanism is directly related to the attachment of the radical species. ISC mechanism may be expected if the spin bearing units are connected through spin 95 nodal sites to high spin bearing positions of the linker in the photo-excited $T_{1}$ state. Since the positions adjacent to the silicon atom are the best spin populated ones in the $T_{1}$ state, ${ }^{16}$ TPSNN and TPSIN (Figure 2) were designed and synthesized by considering the above-mentioned requirements.

100

Synthesis of TPSNN and TPSIN

TPSNN was prepared following the synthetic methodology reported in scheme $1 .{ }^{20}$ It involves a cross coupling reaction between organozinc derivative 2 and the $\mathrm{Me}_{3} \mathrm{Si}$-protected 105 dihydroxylamine $\mathbf{4}$, followed by acidic hydrolysis allowing the removal of the protecting groups. Oxidation of $\mathbf{5}$ by phase transfer reaction using sodium periodate in water / dichloromethane afforded the crude TPSNN diradical as a very hygroscopic deep green solid in $78 \%$ yield. 110 Unfortunately TPSNN was unstable either in concentrated dichloromethane solutions or in the solid state. Concentrated TPSNN solutions yield a mixture of products in which crystallizes a white compound that has been clearly identified as a iso-butylammonium salt. Some examples of 115 nitronylnitroxide photochemical degradation have already been reported by Ullman et al., ${ }^{21,22}$ but in all cases the 2,3dimethylbutane pattern has been identified in the products. To our knowledge, the only skeleton rearrangement that may be invoked to explain the formation of iso-butylamine has been 120 proposed to support the fragmentation mechanism of nitronylnitroxide radicals in electrospray mass spectrometry experiments. $^{23}$ As shown in Scheme 2, the fragmentation pathway upon electrospray ionization possibly involves the reductive removal of the oxygen atoms followed by a
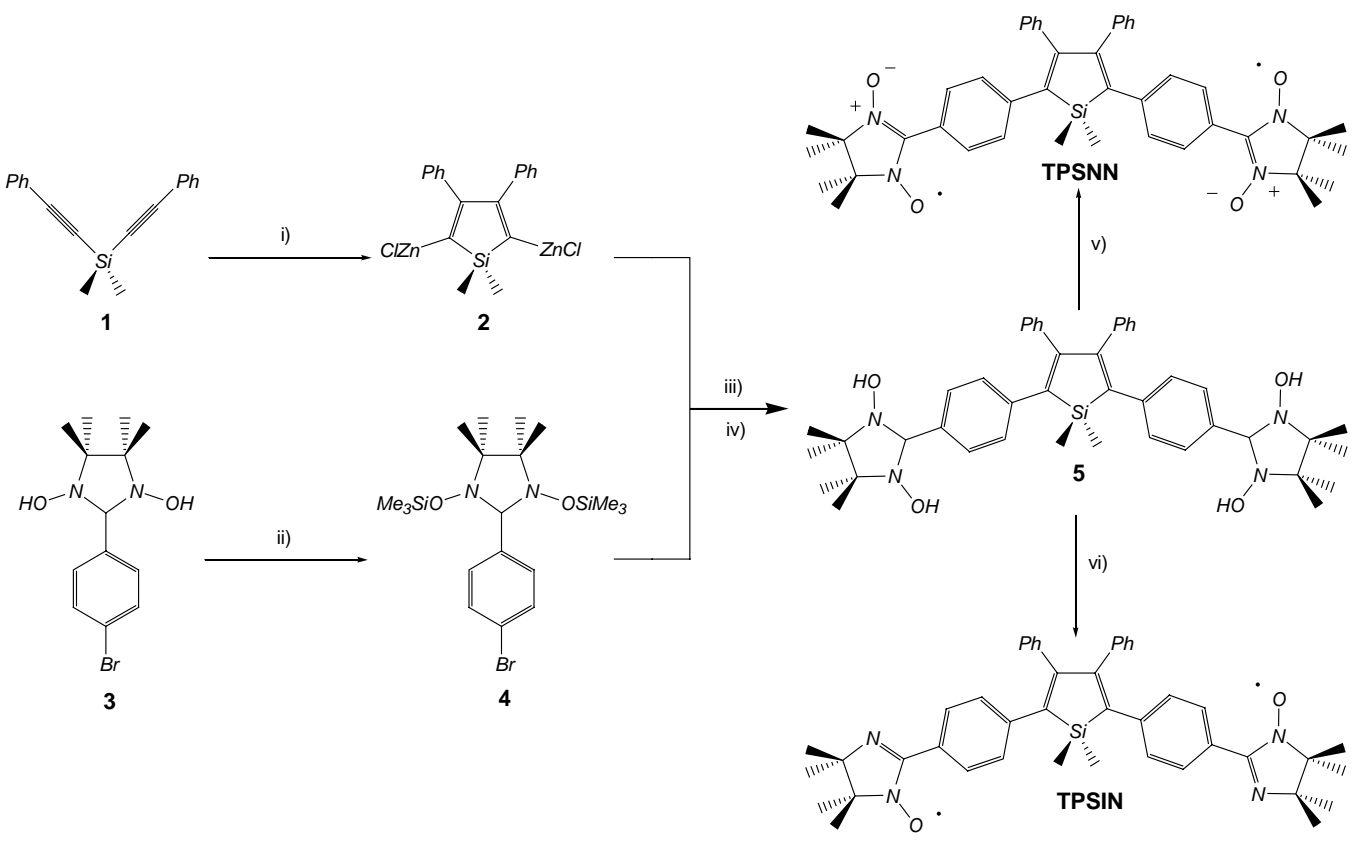

Scheme 1. i) $4 \mathrm{~Np} / \mathrm{Li}$, THF; $4 \mathrm{ZnCl}_{2}$-TMEDA; ii) $3 \mathrm{Me}_{3} \mathrm{SiCl}, 3 \mathrm{Et} 3 \mathrm{~N}$, THF; iii) $\mathrm{PdCl}_{2}\left(\mathrm{PPh}_{3}\right)_{2}$, THF; iv) $\mathrm{HCl} 0.1 \mathrm{M}$; v) $\mathrm{NaIO}_{4}, \mathrm{CH}_{2} \mathrm{Cl}_{2} / \mathrm{H}_{2} \mathrm{O}$; vi) $\mathrm{NaNO}_{2}$, AcOH, $\mathrm{CH}_{2} \mathrm{Cl}_{2}, \mathrm{H}_{2} \mathrm{O} ; \mathrm{NaIO}_{4}, \mathrm{CH}_{2} \mathrm{Cl}_{2} / \mathrm{H}_{2} \mathrm{O}$. 


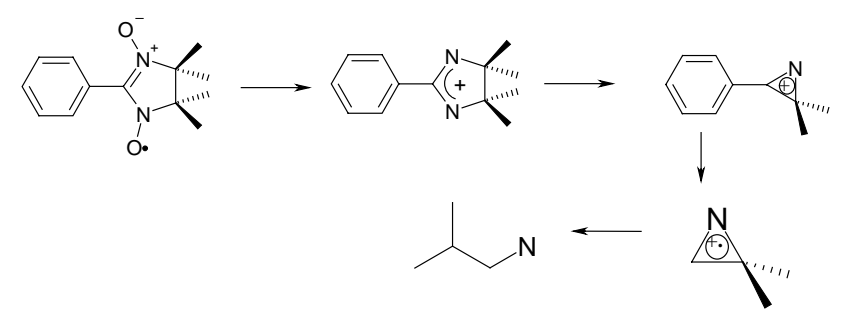

Scheme 2 Proposed electrospray ionization-induced fragmentation mechanism for nitronyl nitroxide radicals after. ${ }^{23}$

125 rearrangement of the transient tetramethylimidazolium cycle into a dimethylaziridinium cation. The further cleavage of the benzylic bond yields a dimethylaziridinium radical cation that may experience a ring-opening transformation to afford the observed iso-butylamine. It is worthy of note that we have no 130 evidence for the validity of this mechanism in our case, but it has the merit to explain this very unexpected fragmentation. TPSIN has been prepared in good yield by treatment of $\mathbf{5}$ with sodium nitrite in slightly acidic conditions followed by oxidation with sodium periodate. In contrast to TPSNN, the

135 bisimononitroxide radical TPSIN that is stable both in the solid state and in concentrated solution, was purified by

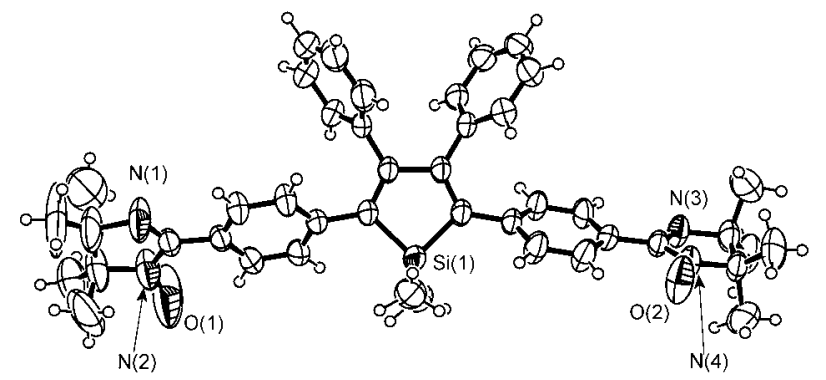

Fig. 3 ORTEP view of the molecular structure of TPSIN (thermal ellipsoids set at $50 \%$ of probability).

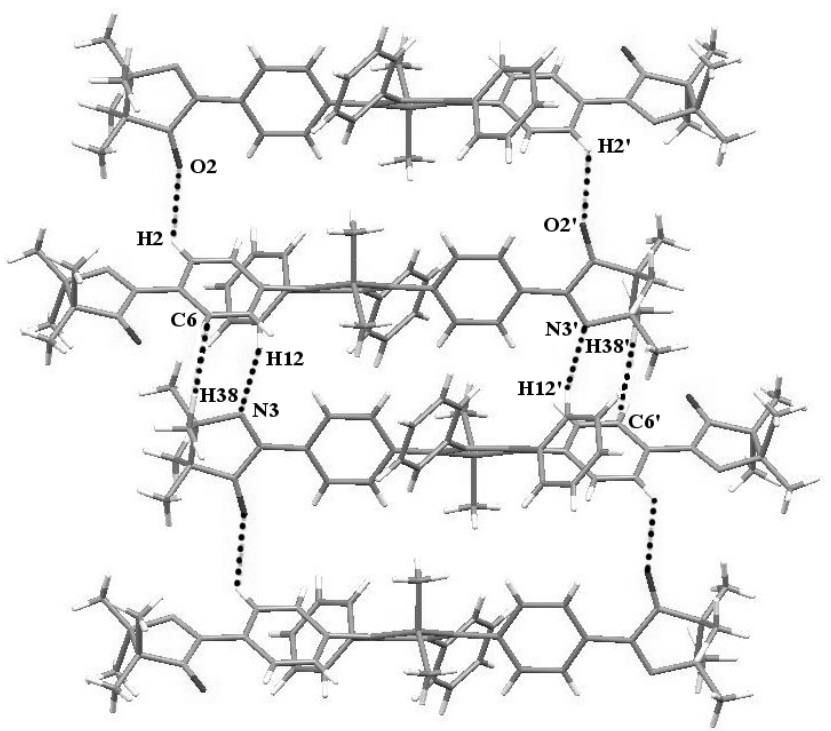

Fig. 4 Side view of a dimer, showing NO $\cdots \mathrm{H}$ weak hydrogen bonds (dashed lines) and additional $\mathrm{N} \cdots \mathrm{H}$ weak hydrogen bonds yielding a stairlike arrangement of the dimers to form infinite supramolecular chains.

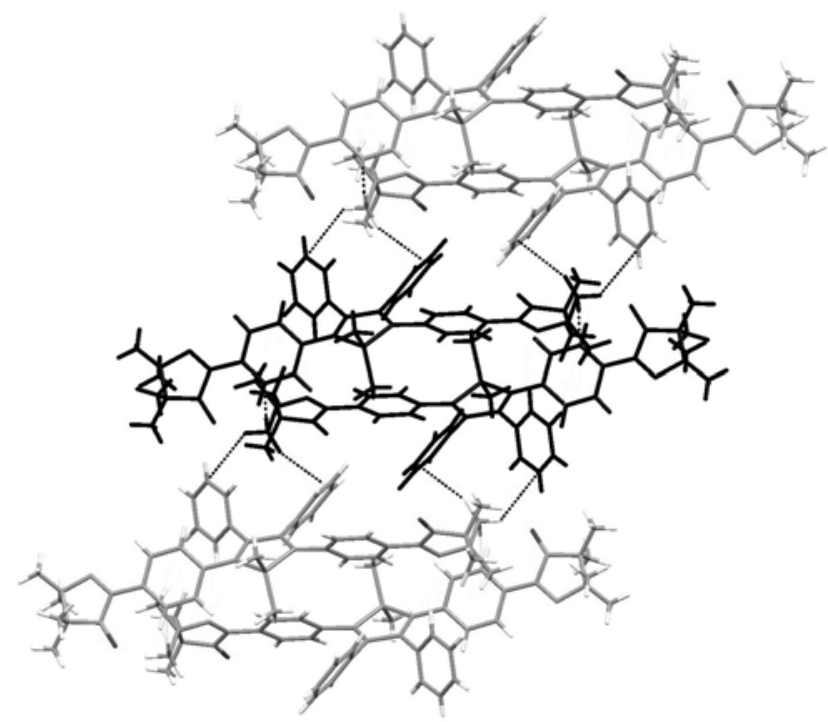

Fig. 5. Top view of a dimer (in black) interacting through C38-H38 $\cdots \pi$ interactions (dashed lines) with two siloles belonging to two different dimers.

preparative TLC. Orange spearhead monocrystals suitable for $\mathrm{X}$-ray analysis were obtained by slow diffusion from a dichloromethane solution of TPSIN layered with pentane.

140

\section{$\mathrm{X}$-ray structure description}

TPSIN crystallizes in the $\mathrm{P}_{-1}$ triclinic space group with two molecules of TPSIN packed in the unit cell. An ORTEP view is presented in Figure 3. The silole displays a propeller-like 145 arrangement of the four benzene rings as usually observed with the others tetraarylsiloles. The dihedral angles between the mean plane of the central silole and the 2,5-benzene rings bearing the nitroxide radicals have values of $54.2(1)^{\circ}$ and $59.8(1)^{\circ}$ which are in the range of those previously reported 150 for related siloles. The two iminonitroxide rings are nearly coplanar with the 2,5-phenyl rings and make with them dihedral angles of $2.3(1)^{\circ}$ and $7.4(1)^{\circ}$ respectively. The $\mathrm{N}$ $\mathrm{O} \cdots \mathrm{N}-\mathrm{O}$ intramolecular distance is of 15.14(3) $\AA$.

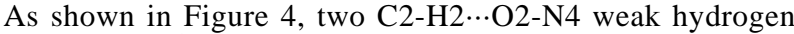
155 bonds $\left(2.51(2) \AA, 134.2(1)^{\circ}\right)$ lead to the formation of a head to tail silole dimer. ${ }^{24,25}$ The other nitrogen atoms of the same iminonitroxide groups are involved in the formation of two C12-H12N3 weak hydrogen bonds $\left(2.64(2) \AA, 164.8(1)^{\circ}\right)$, that are connecting the dimers together with C38${ }_{160} \mathrm{H} 38 \mathrm{~A} \cdots \pi$ interactions with the phenyl rings connected to $\mathrm{C} 2$ to form infinite supramolecular stair-like tapes. ${ }^{26}$ It is worthy to note that $\mathrm{N} 1$ and $\mathrm{N} 2$ containing iminonitroxide groups are not involved in any supramolecular interaction with neighbouring molecules and that the shortest $\mathrm{N}-\mathrm{O} \cdots \mathrm{N}-\mathrm{O}$ 165 distance (6.78(2) $\AA$, N2-O1 $\cdots \mathrm{N} 4-\mathrm{O} 2)$ is observed in a dimer. Packing of the tapes is assumed through C38-H38B $\cdots \pi$ and C38-H38C $\cdots \pi$ interactions with phenyl rings in 3,4-positions (four by molecule, see figure 5).

${ }_{170}$ UV-Visible absorption spectra for siloles 5, TPSNN and TPSIN.

The UV-visible spectra of these compounds have been measured in chloroform. Their data are summarized in Table 1. In the UV-Visible absorption spectra of siloles, it is known 175 that the absorption maximum, ascribed to the $\pi-\pi^{*}$ transition of the 2,5-diarylsilole $\pi$-conjugated moieties, significantly 
depends on the nature of the 2,5-diaryl groups and on the nature and the position of the substituents on them. The $\lambda_{\max }$ ascribed to the $\pi-\pi^{*}$ transition vary from the UV region to the Table 1 UV-visible absorption spectral data for diradicals 5, 6 and 7. ${ }^{a, b}$

\begin{tabular}{cccc} 
Silole & $\pi \rightarrow \pi^{*}{ }_{\text {Ar-NO }}$ & $\pi \rightarrow \pi^{*}$ Silole & $n \rightarrow \pi^{*}{ }_{\text {No }}$ \\
\hline $\mathbf{5}$ & - & $372(5.05)$ & - \\
TPSNN & $262(5.47)$ & $375(5.16)$ & $603(3.79)$ \\
TPSIN & $258(4.27)$ & $370(3.93)$ & $483(2.84)$ \\
\hline
\end{tabular}

${ }^{a} 10^{-3} \mathrm{M}$ solutions in $\mathrm{CHCl}_{3}{ }^{\mathrm{b}} \lambda_{\max }$ in $\mathrm{nm},(\log \varepsilon)$.

180 visible region. For TPSNN and TPSIN, the formation of the diradical species is accompanied by the appearance of two absorption bands ascribed to the Ar-NO $\pi-\pi^{*}$ transitions and to the $\mathrm{N}-\mathrm{O} n-\pi^{*}$ transitions, respectively. Small variations are observed for the siloles $\pi-\pi^{*}$ transitions, indicating weak 185 differences in electronic effects between the parent dihydroxylamine $\mathbf{5}$ and the corresponding imino and nitronylnitroxide radicals.

\section{ESR spectra of TPSIN}

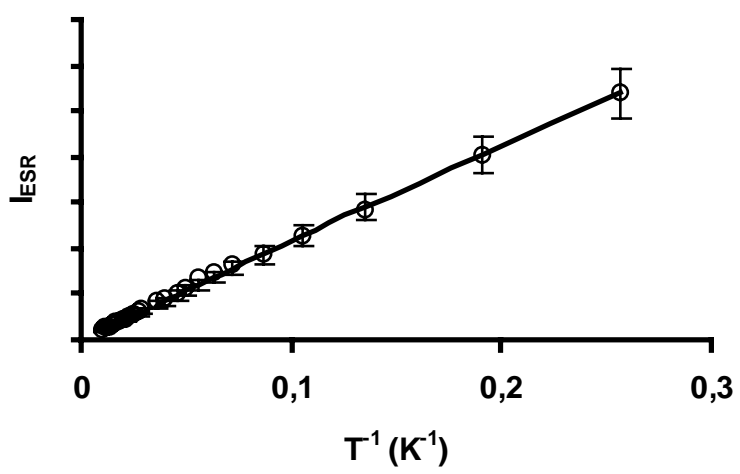

Figure 6. Temperature dependence of the ESR signal intensities of the $\Delta M_{\mathrm{S}}=2$ transition for diradical TPSIN.

190 ESR spectra of the nitroxide diradical TPSIN in degassed dichloromethane solutions were measured in the range 4-298 K. At $298 \mathrm{~K}$, it shows a characteristic thirteen lines spectrum due to hyperfine coupling between the two non-equivalent pairs of nitrogen atoms. Hyperfine coupling constants were 195 determined to be $\mathrm{a}_{\mathrm{N} 1} / 2=4.61 \mathrm{G}$ and $\mathrm{a}_{\mathrm{N} 2} / 2=2.26 \mathrm{G}$, respectively. The spectrum is in agreement with a nitroxide diradical in which the exchange coupling parameter $J$ is substantially larger than the nitrogen hyperfine coupling $\left(|J|>>\mathrm{a}_{\mathrm{N}}\right)$. At $4 \mathrm{~K}$, ESR spectra gave broad signals due to

$$
I_{E S R}=\frac{C}{T}\left[\frac{1}{3+\exp \left(\frac{\Delta E_{\mathrm{T}-\mathrm{s}}}{k_{B} T}\right)}\right]
$$

200 weak dipolar coupling of the unpaired electrons including $\Delta \mathrm{m}_{\mathrm{s}}$ $=2$ transitions at about $1715 \mathrm{G}$. To determine the nature of the magnetic interactions, the intensity of the half-field transition was measured as a function of the temperature $(4-30 \mathrm{~K})$. As the temperature was elevated, the signal due to the triplet

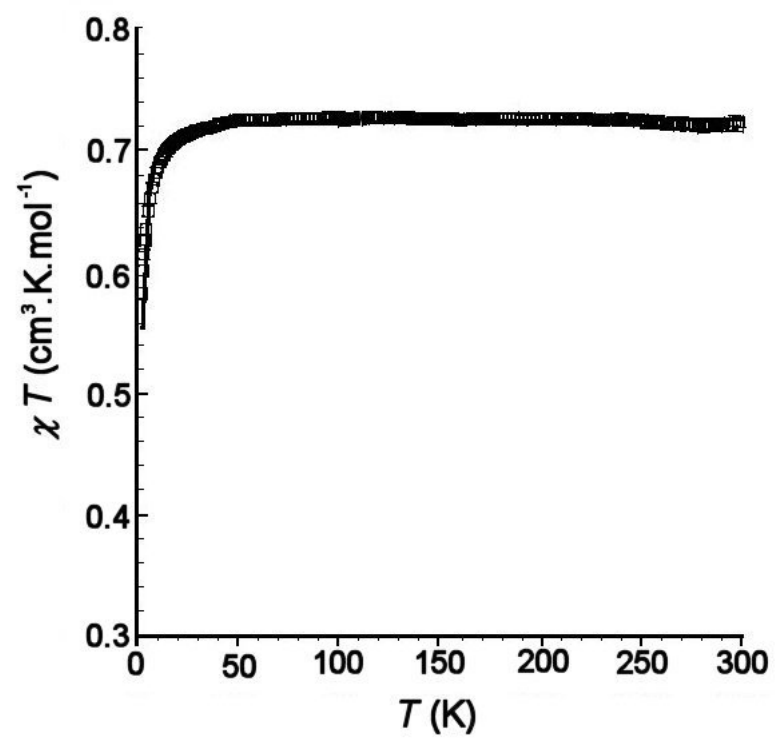

Figure 7. Temperature dependence of the molar magnetic susceptibility $\left(\chi_{\text {mol }}\right)$ as expressed by $\chi_{\text {mol }} T$ vs $T$ plots for TPSIN. The experimental data (open squares) and the theoretical behaviour (solid line, see text) are overlapping.

205 increased in intensity in accordance with the Curie law, indicating that both the singlet ground state and the thermally populated triplet state are nearly degenerated in TPSIN diradical (Figure 6).

210 The best data fit according to the Bleaney-Bowers model gives a singlet-triplet energy gap $\Delta E_{\mathrm{T}-\mathrm{S}} / \mathrm{k}_{\mathrm{B}}$ of $0.9 \mathrm{~K}$, using $\mathrm{Eq}$ 1 to describe the Boltzmann distribution between the two states where $\mathrm{C}$ is a proportionality constant.

\section{Magnetic susceptibility of TPSIN}

The static magnetic susceptibility of a polycrystalline sample of TPSIN was measured in the range 2-300 K with a SQUID susceptometer at a constant magnetic field of 5000 Gauss. The temperature dependence of the molar magnetic susceptibility

${ }_{220}\left(\chi_{\mathrm{mol}}\right)$ is shown in the form $\chi_{\mathrm{mol}} T$ vs $T$ in Figure 7 . The observed $\chi_{\mathrm{mol}} T$ value is $0.72 \mathrm{~cm}^{3} \mathrm{~K} \mathrm{~mol}^{-1}$ at $300 \mathrm{~K}$, suggesting that the singlet and the triplet states are nearly statistically populated at ambient temperature and that a slight amount of monoradical is present in the sample. However, the

$225 \chi \cdot T$ product value is in good agreement with the theoretical value of $0.75 \mathrm{~cm}^{3} \cdot \mathrm{K} \cdot \mathrm{mol}^{-1}$ expected for two uncorrelated $\mathrm{S}=1 / 2$ units with $\mathrm{g}=2.0$.

$$
\chi_{m o l}=f \cdot \frac{2 N g^{2} \mu^{2} B}{k_{B} T} \cdot \frac{1}{3+\exp \left(-2 J / k_{\mathrm{B}} T\right)}
$$

On decreasing the temperature, the $\chi_{\text {mol }} T$ value remains 230 almost constant in the temperature range $300-10 \mathrm{~K}$, and then decreases to reach $0.58 \mathrm{~cm}^{3} . \mathrm{K} \cdot \mathrm{mol}^{-1}$ as the temperature is lowered down to $2 \mathrm{~K}$. The temperature dependence of the $\chi_{\text {mol }} T$ value was analysed in terms of a modified singlettriplet two state model where the magnetic exchange coupling 235 constant $J$ corresponds to an Hamiltonian of the form $H=-$ $2 J S_{I} S_{2}$. A purity factor $f$ was introduced for microcrystalline samples of the diradical used for the magnetic 
measurements. ${ }^{27}$ The best fit parameters were $J / k_{B}=-1.0 \mathrm{~K}$, and $f=0.96$ (Eq 2).

240

\section{Time-resolved ESR experiments}

Since the existence of a photo-accessible triplet state for the silole itself remained elusive, we measured the photo-excited state of the parent TPS by time-resolved ESR. A typical

245 TRESR spectrum of the parent TPS is shown in Figure 8(a). The observed TRESR spectrum with well-resolved fine structure splitting has been unambiguously analysed to be an excited triplet state by the spectral simulation shown in Figure 8(b). The determined $g$ value, fine-structure parameters, and 250 relative populations of the $M s$ sublevels are listed in Table 2.

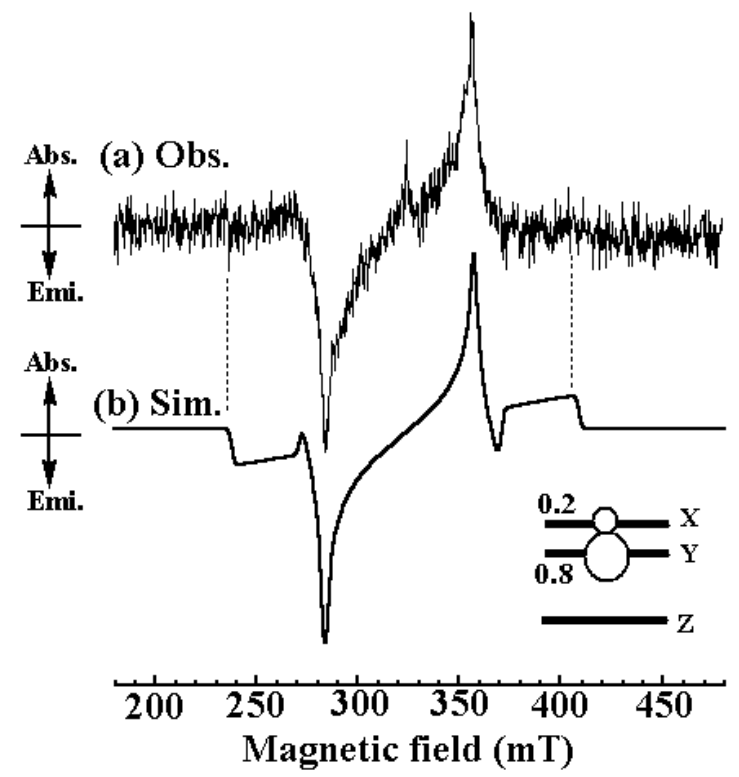

Figure 8. Time resolved ESR of TPS in EPA rigid glass $0.4 \mu \mathrm{s}$ after a Nd:YAG pulse laser excitation $(\lambda=355 \mathrm{~nm})$. (a) Observed spectrum at 30 K. (b) Simulation. The spin Hamiltonian parameters used in the simulation are described in Table 2.

The observed TRESR spectrum shows the $E / A$ pattern of the

Table 2. Spin Hamiltonian parameters and relative polarization of $M \mathrm{~s}$ sublevels in zero-field for TPS.

\begin{tabular}{ccc}
\hline g value & Fine-Structure Parameters & $\begin{array}{l}\text { Relative } \\
\text { Polarizations }\end{array}$ \\
\hline 2.010 & $D=0.076 \mathrm{~cm}^{-1}, E=0.002 \mathrm{~cm}^{-1}$ & $\begin{array}{c}P_{\mathrm{X}}=0.2, P_{\mathrm{Y}}=0.8 \\
P_{\mathrm{Z}}=0.0\end{array}$ \\
\hline
\end{tabular}

dynamic electron polarization (DEP) (eeaa) (e/a: emission/absorption of microwave). This indicates that a selective intersystem crossing by the spin-orbit interaction 255 (SO-ISC) occurs toward the spin-sublevel, $Y$, of the zero-field wave-functions. Despite multiple attempts to observe TRESR signals characteristics of high-spin photo-excited states for TPSIN, no signal was obtained studying this diradical in contrast to the results reported when 9,10-diphenylanthracene 260 was used as a spin coupler. ${ }^{8,10,11,13}$

\section{Discussion.}

Structurally speaking, diradical TPSIN belongs to a class of diradicals termed as doubly disjoint by Lahti et al. ${ }^{28}$ It is 265 mainly constituted by a non-alternating heteropentacyclic system (the silole ring) in which spin bearing units are connected to the central ring through sites with low spin density: weak antiferromagnetic interactions and nearly degenerated singlet and triplet states are predicted from both 270 valence-bond and spin polarization theory for this kind of molecule. ${ }^{29}$ The intramolecular distance between the two radical centers is greater than $15 \AA$. In dilute solutions, this distance is too remote to allow through-space intramolecular magnetic interactions. ${ }^{30}$ As a consequence, the magnetic 275 interactions observed following the half field signal intensity by ESR traduce the existence of very weak antiferromagnetic interactions that are propagated through the skeleton of the molecule $\left(J_{E S R}=-1 / 2\left(\Delta E_{\mathrm{T}-\mathrm{S}} / \mathrm{k}_{\mathrm{B}}\right) \approx-0.5 \mathrm{~K}\right)$. The slight difference observed between the $J_{S Q U I D}(-1.0 \mathrm{~K})$ and $J_{E S R}$ values may

280 traduce the existence of weak additional antiferromagnetic intermolecular interactions in the solid state. The $\mathrm{N}-\mathrm{O} \cdots \mathrm{N}-\mathrm{O}$ shortest distances observed in a dimer $(6.78 \AA$ ) are too remote to allow short contact type magnetic interactions. Magnetic interactions may be expected through hydrogen bonding

285 considering $\mathrm{C}-\mathrm{H} \cdots \pi$ interactions and $\mathrm{C}-\mathrm{H} \cdots \mathrm{N}$ or $\mathrm{C}-\mathrm{H} \cdots \mathrm{O}-\mathrm{N}$ hydrogen-bonded motives. The well known low spin density delocalisation on methyl groups in imino or nitronylnitroxide radicals together with the low spin density delocalisation on the 3,4-phenyl rings allow to discard this hypothesis. ${ }^{31}$

290 Another way to explain such a slight difference is to consider that in the solid state the molecular conformation of TPSIN allows better magnetic exchanges than in frozen solution, in which numerous and less favourable conformations are possible. Whatever the explanation, $J$ values are in agreement 295 with the values ascribed to disjoint systems, with the values encountered in the case of TPSNO $(-4 \mathrm{~K})$ and with the ones obtained when diphenylanthracene is used as a coupler $(-2.9$ K). ${ }^{11,17,18}$

Turning back to TRESR experiments, we can consider the 300 following reasons to explain the lack of high-spin photoexcited states in the case of silole-based diradicals: (1) the life-time of the triplet state of the parent silole (TPS) is much shorter than the ones observed for anthracene derivatives (the triplet life-time of anthracene is $c a$. $55 \mathrm{~ms}$ and the triplet life305 time of TPS is about few $\mu \mathrm{s}$ ), and (2) the efficiency of the enhanced SO-ISC is much smaller than the one encountered in diphenylanthracene-bisiminonitroxide. Concerning the last point, the SO-ISC efficiency highly depends of the nature of the spin bearing units and of the electronic structure of the 310 triplet photo-excited spin coupler. Since TPSIN has been designed to present the appropriate topology, and the molecule is built with a coupler that presents both a triplet photo-excited state and iminonitroxide radicals, it is reasonable to postulate that the enhanced SO-ISC mechanism 315 is also operating in the present diradical. Therefore, the only way to explain the lack of high-spin photo-excited state is to consider that the strong non-radiative relaxation processes from the triplet spin coupler make the triplet lifetime shorter, leading to the unsuccessful detection of high-spin photo320 excited states within the time-scale of TRESR measurement. Both factors arising from the molecular and electronic structures prevent the effective generation of the expected result. Therefore it is of crucial importance that the $\pi$ conjugated spin coupler pocesses a very rigid skeleton to 325 restrict to a minimum the non-radiative deactivition channel 
that may be opened if sets of molecular motion or vibrations are available. This is why rigid fused polyaromatic structures such as fullerene, porphyrine, pyrene, and of course, anthracene, are used to achieve photo-excited spin alignment.

\section{${ }_{330}$ Conclusions}

In this article we have reported the synthesis of two nitroxidebased diradicals linked by a 2,3,4,5-tetraphenylsilole (TPS) pattern. These two molecules have been especially designed and synthesized with the aim to access to high-spin photo335 excited states for both of these species. Whereas the bisiminonitroxide diradical TPSIN is stable either in solution or in the solid state, its correponding bisnitronylnitroxide TPSNN experiences a spontaneous fragmentation to afford a iso-butylammomium salt. Magnetic behaviour of TPSIN has

340 been analysed by means of ESR and SQUID measurements combined with structural considerations. As expected from its doubly disjoint character, the TPSIN diradical displays weak intramolecular antiferromagnetic interactions with $J / k_{B}=-1.0$ $\mathrm{K}$. Although the combination of the silole core with 345 iminonitroxide radicals would be expected to lead to photoinduced spin alignment and to high spin states, no detection of high-spin states was possible within the time-scale of TRESR measurement. Therefore, TPS-derivatives clearly provide evidences that besides the needs of a photo-tunable coupler,

350 specific radical species, and $\pi$-topological requirements, the flexibilty of the coupler skeleton also plays an important role in the achievement of photo-excited high spin states. Taking into account that the silole ring, with its unique electronic structure and its triplet photo-excited state, is all the same a

355 good candidate to achieve the photo-induced spin alignment, synthetic works are currently under progress to increase the rigidity of the whole structures by fusing the spin-bearing units to the central organometallic linker.

\section{${ }_{360}$ Experimental}

\section{Materials and methods}

The photo-excited states of the siloles were examined by TimeResolved ESR (TRESR) at the Department of Material Science of the Osaka City University. A conventional X-band ESR spectrometer 365 (JEOL TE300) was used in the measurements of TRESR spectra without field modulation. Excitation was carried out at $355 \mathrm{~nm}$ light using Nd:YAG pulse laser (Continuum Surelite II-10, pulse width $<7$ ns). The typical laser power used in the experiments was c.a. $2 \sim 5$ mJ. EPA glass matrix was used for the TRESR experiments. The

370 measurements were carried out at $30 \mathrm{~K}$. The typical microwave power was $c a$. $10 \mathrm{~mW}$. The spectral simulation was carried out by the eigenfield/exact-diagonalization hybrid method, ${ }^{32-33}$ taking dynamic electron spin polarization (ESP) into account. The following ordinary spin Hamiltonian given in eq. (3) was used for the analysis.

$375 \quad \mathrm{H}_{\text {spin }}^{\prime}=\beta_{\mathrm{e}}$ H.g.S + S.D.S

The resonance field $B_{M s \leftrightarrow M s+I}(\theta, \phi)$ for each transition was directly calculated by the eigenfield method. ${ }^{32}$ The transition probabilities $I$ $(\theta, \phi, \varphi)$ were evaluated by numerically diagonalization of the spin Hamiltonian matrix at the calculated resonance field. Since the

380 resonance field is independent of the third Euler angle, $\varphi$, the above procedure practically saves the computing time for the simulation. The line-shape function of the TRESR spectrum in the glass matrix is given by $\mathrm{g}(\mathrm{B})=\mathrm{N} \Sigma \int \mathrm{d} \varphi \int \mathrm{d} \phi \int \mathrm{d} \theta \sin \theta \mathrm{P}_{\mathrm{Ms} \leftrightarrow \mathrm{Ms}+1}(\theta, \phi) \quad \mathrm{I} \quad(\theta, \phi, \varphi) \quad \mathrm{f}[\mathrm{B}-$ $\left.385 \mathrm{~B}_{\mathrm{Ms} \leftrightarrow \mathrm{Ms}+1}(\theta, \phi)\right]$

In the simulation, the dynamic electron polarization (ESP), $P_{M s \leftrightarrow M s+1}(\theta, \phi)$, on each spin sublevel in a zero magnetic field was given as parameters. Thus, the relative populations of the Ms sublevels were taken into account as parameters. The simulation was
390 carried out using a program written by the author on a personal computer. The details of the simulation procedures for the high-spin TRESR spectra were described in our previous articles.

${ }^{1} \mathrm{H},{ }^{13} \mathrm{C}$ and ${ }^{29} \mathrm{Si}$ NMR spectra were recorded on a Bruker Advance 395200 DPX spectrometer, the FT-IR spectra on a Thermo Nicolet Avatar 320 spectrometer, the UV-visible spectra on a Secomam Anthelie instrument and the MS spectra on a Jeol JMS-DX 300 spectrometer. The ESR spectra have been recorded on X-band Bruker Elexsys spectrometer. Magnetic susceptibility measurements were 400 obtained with a Quantum Design MPMS-5S SQUID magnetometer. All reactions were carried out routinely under nitrogen using standard Schlenck techniques. Solvents were distilled prior to use. THF was dried over sodium/benzophenone, and distilled under Argon. All the commercial reagents were used as received. 405 Bis(phenylethynyl)dimethylsilane was prepared by the reaction of dimethyldichlorosilane and phenylethynyllithium, which was prepared from $n \mathrm{BuLi}$ and phenylacetylene in ether.

\section{Syntheses}

410 1,3-Bis(trimethylsilyloxylamino)-2-(4-bromophenyl)-4,4,5,5tetramethylimidazolidine (4). To a solution of 1,3-Dihydroxy-2-(4bromophenyl)-4,4,5,5-tetramethylimidazolidine ${ }^{34}$ (3.15 g, $10 \mathrm{mmol}$ ) in THF $(30 \mathrm{~mL})$ was added an excess of triethylamine $(8.3 \mathrm{~mL}, 60$ $\mathrm{mmol})$. To the reaction mixture was added chlorotrimethylsilane 415 (7.60 mL, $60 \mathrm{mmol})$ in THF $(50 \mathrm{~mL})$. After stirring at $45^{\circ} \mathrm{C}$ for $20 \mathrm{~h}$, the solvents were evaporated to yield a residue that was treated with $200 \mathrm{~mL}$ of pentane and filtered. The filtrate was then evaporated in vaccuo to yield $1.92 \mathrm{~g}$ of 4 as white crystals (42\%). M.p.: $87-89^{\circ} \mathrm{C}$. IR $\left(\mathrm{CHCl}_{3}, \mathrm{~cm}^{-1}\right): 1373\left(\mathrm{v}_{\mathrm{N}-\mathrm{O}}\right) .{ }^{1} \mathrm{H}$ NMR $\left(\mathrm{CDCl}_{3}, 25^{\circ} \mathrm{C}\right): \delta=-0.22$ (s, $42018 \mathrm{H}), 1.18(\mathrm{~s}, 12 \mathrm{H}), 4.60(\mathrm{~s}, 1 \mathrm{H}), 7.32\left(\mathrm{~d},{ }^{3} J_{\mathrm{HH}}=8 \mathrm{~Hz}, 2 \mathrm{H}\right), 7.48(\mathrm{~d}$, $\left.{ }^{3} J_{\mathrm{HH}}=8 \mathrm{~Hz}, 2 \mathrm{H}\right) .{ }^{13} \mathrm{C} \mathrm{NMR}\left(\mathrm{CDCl}_{3}, 25^{\circ} \mathrm{C}\right): \delta=-0.36,16.95,24.20$, 61.06, 92.37, 119.18, 121.49, 130.37, 131.71. ${ }^{29} \mathrm{Si} \mathrm{NMR} \mathrm{(DMSO-d}{ }_{6}$, $25^{\circ} \mathrm{C}$ ): $\delta=22.66$. HRMS (FAB+, m-nitrobenzyl alcohol matrix): $\mathrm{m} / \mathrm{z}$ : calcd for $\mathrm{C}_{19} \mathrm{BrH}_{36} \mathrm{~N}_{2} \mathrm{O}_{2} \mathrm{Si}_{2}\left[\mathrm{M}^{+}+\mathrm{H}\right]$ : 459.1481; found 459.1495.

425

Silole 5. A mixture of lithium $(0.1 \mathrm{~g}, 14.5 \mathrm{mmol})$ and naphthalene $(1.85 \mathrm{~g}, 14.5 \mathrm{mmol})$ in THF $(15 \mathrm{~mL})$ was stirred at room temperature under argon for $5 \mathrm{~h}$ to form a deep green solution of lithiumnaphthalenide. To this mixture was added 430 bis(phenylethynyl)dimethylsilane 1 ( $1 \mathrm{~g}, 3.85 \mathrm{mmol})$ in THF (10 $\mathrm{mL}$ ). After stirring for $10 \mathrm{~min}$, the reaction mixture was cooled to $0^{\circ}$ $\mathrm{C}$ and $\left[\mathrm{ZnCl}_{2}\right.$ (tmen) $]$ (tmen $=N, N, N^{\prime}, N^{\prime}$-tetramethylenediamine) $(3.9$ g, $14.5 \mathrm{mmol}$ ) was added as a solid to form organozinc derivative 2 . After stirring for one hour at room temperature, a solution of 4 (3.5 g, $4357.65 \mathrm{mmol})$ in THF $(10 \mathrm{~mL})$ and $\left[\mathrm{PdCl}_{2}\left(\mathrm{PPh}_{3}\right)_{2}\right](0.14 \mathrm{~g}, 0.2 \mathrm{mmol})$ were successively added. The mixture was heated under reflux and stirred for $24 \mathrm{~h}$. After hydrolysis by acetic acid (35\%), the mixture was extracted with $\mathrm{Et}_{2} \mathrm{O}$ several times. The combined organic layers were washed with brine, saturated solutions of $\mathrm{Na}_{2} \mathrm{CO}_{3}$, dried over $440 \mathrm{MgSO}_{4}$ and concentrated. The resulting residue was subjected to a column chromatography (neutral silica , eluant: pentanedichloromethane $80: 20)$ to give $1.57 \mathrm{~g}$ of $\mathbf{5}$ as a yellow solid (56\%). Dec. temp.: $192^{\circ} \mathrm{C}$. IR $\left(\mathrm{KBr}, \mathrm{cm}^{-1}\right)$ : 3529, $3242\left(v_{\mathrm{O}-\mathrm{H}}\right), 1364\left(v_{\mathrm{N}-\mathrm{O}}\right)$. UV/Vis $\left(\mathrm{CHCl}_{3}\right): \lambda_{\max }(\log \varepsilon): 372\left(5.05, \pi \rightarrow \pi^{*}\right.$ silole). ${ }^{1} \mathrm{H}$ NMR $445\left(\mathrm{CDCl}_{3}, 25^{\circ} \mathrm{C}\right): \delta=0.47(\mathrm{~s}, 6 \mathrm{H}), 1.02(\mathrm{~s}, 12 \mathrm{H}), 1.06(\mathrm{~s}, 12 \mathrm{H}), 4.40(\mathrm{~s}$, $2 \mathrm{H}) 6.83-7.06(\mathrm{~m}, 8 \mathrm{H}), 7.09(\mathrm{~m}, 6 \mathrm{H}), 7.25\left(\mathrm{~d},{ }^{3} \mathrm{~J}_{\mathrm{HH}}=8 \mathrm{~Hz}, 4 \mathrm{H}\right), 7.75$ (s, 4H, OH). ${ }^{13} \mathrm{C}$ NMR ([D $]$ DMSO, $\left.25^{\circ} \mathrm{C}\right): \delta=-2.80,17.87,25.25$, 66.89, 91.05, 126.76, 127.17, 128.48, 128.56, 129.01, 130.20, 138.78, 139.79, 140.32, 141.43, 154.29. ${ }^{29}$ Si NMR ([D 6 DMSO): $\delta=8.05$. 450 HRMS (FAB+, m-nitrobenzyl alcohol matrix): $\mathrm{m} / \mathrm{z}$ : calcd for $\mathrm{C}_{44} \mathrm{H}_{55} \mathrm{~N}_{4} \mathrm{O}_{4} \mathrm{Si}\left[\mathrm{M}^{+}+\mathrm{H}\right]$ : 731.3993; found 731.3995.

TPSNN. To a solution of silole 5 (100 mg, $0.14 \mathrm{mmol})$ in $20 \mathrm{~mL}$ of freshly distilled dichloromethane, was added $\mathrm{NaIO}_{4}(130 \mathrm{mg}, 0.6$ $455 \mathrm{mmol}$ ) as a solution in $20 \mathrm{~mL}$ of distilled water. The mixture was stirred for one hour and the phases were separated. The aqueous phase was extracted with dichloromethane $(3 \times 20 \mathrm{~mL})$. The organic layers were mixed and dried other $\mathrm{MgSO}_{4}$. The solvent was removed under vacuum and the crude product was purified by preparative thin460 layer chromatography (silicagel, eluant: pentane-ethylacetate 60:40) to give $79 \mathrm{mg}$ of TPSNN (58 mmol) as a very hygroscopic deepgreen solid (78\%). M.p.: not determined. IR $\left(\mathrm{KBr}, \mathrm{cm}^{-1}\right): 1363\left(v_{\mathrm{N}-\mathrm{O}}\right)$. 
UV/Vis $\left(\mathrm{CHCl}_{3}\right): \lambda_{\max }(\log \varepsilon): 262\left(5.47, \pi \rightarrow \pi^{*}\right.$ arylnitroxide), 375 (5.16, $\pi \rightarrow \pi^{*}$ silole), 603 (3.79, $\left.\mathrm{n} \rightarrow \pi^{*} \mathrm{~N}-\mathrm{O}\right)$. HRMS (FAB+, m465 nitrobenzyl alcohol matrix): $m / z$ : calcd for $\mathrm{C}_{44} \mathrm{H}_{50} \mathrm{~N}_{4} \mathrm{O}_{4} \mathrm{Si}\left[\mathrm{M}^{+}+3 \mathrm{H}\right]$ :

726.3601; found 726.3594. Chemical degradation in solution. Typically, a solution of TPSNN $(0.100 \mathrm{~g}, 0.14 \mathrm{mmol})$ in dichloromethane $(5 \mathrm{~mL})$ yields white crystals of iso-butylamine within three days at room temperature. The crystals are collected on a 470 fritt, washed with dichloromethane and dried in air. M. p.: $175^{\circ} \mathrm{C}$. IR $\left(\mathrm{KBr}, \mathrm{cm}^{-1}\right)$ : $3060\left(v_{\mathrm{N}-\mathrm{H}}\right), 2922\left(v_{\mathrm{C}-\mathrm{H}}\right), 1572\left(v_{\mathrm{N}-\mathrm{C}}\right) .{ }^{1} \mathrm{H}$ NMR $\left(\mathrm{CDCl}_{3}\right.$, $\left.25^{\circ} \mathrm{C}\right): \delta=1.02\left(\mathrm{~d},{ }^{3} J_{\mathrm{HH}}=6.8 \mathrm{~Hz} 6 \mathrm{H}\right), 1.63-1.75(\mathrm{~m}, 1 \mathrm{H}), 3.25\left(\mathrm{~d},{ }^{3} J_{\mathrm{HH}}\right.$ $=6.5 \mathrm{~Hz} 2 \mathrm{H}), 7.3(\mathrm{~s}, 2 \mathrm{H})$. MS (FAB+, m-nitrobenzyl alcohol matrix): $\left[\mathrm{M}^{+}\right]: m / z: 74(100 \%)$.

TPSIN. To a solution of silole $5(100 \mathrm{mg}, 0.14 \mathrm{mmol})$ in $20 \mathrm{~mL}$ of freshly distilled dichloromethane, was added $\mathrm{NaNO}_{2}$ (38 $\mathrm{mg}, 0.6 \mathrm{mmol}$ ) as a solution in $20 \mathrm{~mL}$ of distilled water. The mixture was stirred and a 480 few drops of acetic acid were added to obtain a pH value near 6 . After 15 minutes, $\mathrm{NaIO}_{4}$ (38 mg, $0.6 \mathrm{mmol}$ ) was added as a solid and the mixture stirred 15 minutes more. The two phases were separated and the aqueous layer was extracted three times with $20 \mathrm{~mL}$ of dichloromethane. The organic layers were mixed and dried other $\mathrm{MgSO}_{4}$. The solvent was

485 removed under vacuum and the crude product was purified by column chromatography (neutral aluminum oxide, eluant: pentanedichloromethane 80:20) to give $38 \mathrm{mg}$ of TPSIN as red crystals (42\%). M.p.: $182^{\circ} \mathrm{C}$. IR $\left(\mathrm{KBr}, \mathrm{cm}^{-1}\right): 1368\left(v_{\mathrm{N}-\mathrm{O}}\right), 1538\left(v_{\mathrm{C}=\mathrm{N}}\right)$. UV/Vis $\left(\mathrm{CHCl}_{3}\right)$ : $\lambda_{\max }(\log \varepsilon): 258$ (4.27, $\pi \rightarrow \pi^{*}$ arylnitroxide), 370 (3.93, $\pi \rightarrow \pi^{*}$ silole),

490483 (2.84, $\left.\mathrm{n} \rightarrow \pi^{*} \mathrm{~N}-\mathrm{O}\right)$. HRMS (FAB+, m-nitrobenzyl alcohol matrix): $m / z$ : calcd for $\mathrm{C}_{44} \mathrm{H}_{51} \mathrm{~N}_{4} \mathrm{O}_{2} \mathrm{Si}\left[\mathrm{M}^{+}+3 \mathrm{H}\right]$ : 695.3770; found 695.3770 . Experimental and crystal data for TPSIN. Spearhead single crystals of approximate dimensions $0.15 \times 0.08 \times 0.08 \mathrm{~mm}^{3}$ were selected on polarized microscope and mounted on a Bruker-Nonius $\kappa$-CCD

495 diffractometer, Mo- $\mathrm{K}_{\alpha}$ radiation $(0.71073 \AA$ ). Data collection was performed using mixed $\phi$ and $\omega$ scans, 179 frames of $1.5^{\circ}, 285$ seconds per frame and a distance crystal-detector of $30 \mathrm{~mm}$. The structural determination by direct methods and the refinement of atomic parameters based on full-matrix least squares on $F^{2}$ were performed using the 500 SHELX-97. ${ }^{35-36}$ Results: $a=10.984(1) \AA, b=11.474(1) \AA, c=17.492(1)$ $\AA, \alpha=81.10(1)^{\circ}, \beta=89.01(1)^{\circ}, \gamma=65.70(2), V=1982.6(9) \AA^{3}$, density (calc.) $=1.161$, triclinic P-1, $97.7 \%$ completeness to theta $26.48^{\circ}$, 12809 collected data, 8110 independent reflections $\left(R_{\text {int }}=0.032\right)$ for 605 refined parameters, $R_{\mathrm{obs}}=0.069, w R_{\text {obs }}^{2}=0.147,(\Delta / \sigma)_{\max }=0.001$, largest 505 difference peak and hole $0.46 /-0.42$ e. $\mathrm{A}^{-3}$, max. CCDC - 603216 contains the supplementary crystallographic data for this paper. These data can be obtained free of charge from The Cambridge Crystallographic Data Centre via www.ccdc.cam.ac.uk/datarequest/cif.

\section{${ }_{510}$ Acknowledgments}

This work was supported by the French CNRS, the European Commission through the Network of Excellence MAGMANet (NMP3/CT/2005/515767), and the Grant-in-Aid for Scientific Research on Priority Area "Application of Molecular Spin” 'Area 515 769, Prop. No. 15087208) from MEXT, Japan. Ph. G. and N. R. want to warmly thank Prof. Philippe Turek (Université Louis Pasteur, Strasbourg, France), and Prof. Dominique Luneau (Université Claude Bernard-Lyon I, Lyon, France) for their assistance in magnetic characterization and fruitful scientific discussions.

\section{Notes and references}

O. Kahn, 'Molecular Magnetism', ed. VCH, 1993.

P. M. Lahti, 'Magnetic properties of organic materials', Marcel Dekker, Inc., 1999.

R. Ziessel, C. Stroh, H. Heise, F. Khöler, P. Turek, N. Claiser, M. Souhassou, and C. Lecomte, J. Am. Chem. Soc., 2004, 126, 12604.
O. Benedi Borodia, P. Guionneau, H. Heise, F. H. Khöler, L. Ducasse, J. Vidal-Gancedo, J. Veciana, S. Golhen, L. Ouahab, J.-P. Sutter, Chem. Eur. J., 2005, 11, 128.

A. Ito, Y. Nakano, M. Urabe, T. Kato, and K. Tanaka, J. Am. Chem. Soc., 2006, 128, 2948.

O. Sato, S. Hayami, Y. Einaga, and Z.-Z. Gu, Bull. Chem. Soc. Jpn., 2003, 76, 443.

Y. Teki, T. Toichi, and S. Nakajima, Chem. Eur. J., 2006, 12, 2329.

Y. Teki, Polyhedron, 2001, 20, 1163.

Y. Teki, M. Kimura, S. Narimatsu, K. Ohara, and K. Mukai, Bull. Chem. Soc. Jpn., 2004, 77, 95.

Y. Teki, S. Miyamoto, K. Imura, M. Naktsuji, and K. Miura, J. Am. Chem. Soc., 2000, 122, 984

Y. Teki, S. Miyamoto, M. Naktsuji, and Y. Miura, J. Am. Chem. Soc., 2001, 123, 294

Y. Teki and S. Nakajima, Chem. Lett., 2004, 33, 1500.

Y. Teki, M. Nakatsuji, and Y. Miura, Mol. Phys., 2002, 100, 1385.

C. Corvaja, M. Maggini, M. Prato, G. Scorrano, and M. Venzin, J. Am. Chem. Soc., 1995, 117, 8857.

K. Ishii, J. Fujisawa, Y. Ohba, and S. Yamauchi, J. Am. Chem. Soc., 1996, 118, 13079.

N. Roques, P. Gerbier, S. Nakajima, Y. Teki, and C. Guérin, J. Phys. Chem. Solids, 2004, 64, 759.

N. Roques, P. Gerbier, J.-P. Sutter, P. Guionneau, D. Luneau, and C. Guérin, Organometallics, 2003, 22, 4833.

N. Roques, P. Gerbier, U. Schatzneider, J.-P. Sutter, P. Guionneau, J. Vidal-Gancedo, J. Veciana, E. Rentschler, and C. Guérin, Chem. Eur. J., 2006, published on Web, DOI: 10.1002/chem.200501280.

Y. Teki, Polyhedron, 2005, 24, 2299.

S. Yamaguchi, T. Endo, M. Uchida, T. Izumizawa, K.

Furukawa, and K. Tamao, Chem. Eur. J., 2000, 6, 1683.

E. F. Ullman, L. Call, and S. S. Tseng, J. Am. Chem. Soc., 1975, 75, 1677.

L. Call and F. E. Ullmann, Tetrahedron Lett., 1971, 42, 3935.

C. Smith, J. Bartley, and S. Bottle, J. Mass Spectrom., 2002, 37, 897.

G. Desiraju, 'Crystal engineering: The Crystal as a Supramolecular Entity: Perspectives in Supramolecular Chemistry', Wiley, 1996.

G. Desiraju, Chem. Commun., 2005, 2995.

M. Nishio, Cryst. Eng. Comm., 2004, 6, 130.

T. Matsumoto, T. Ishida, N. Koga, and H. Iwamura, J. Am. Chem. Soc., 1992, 114, 9952.

P. M. Lahti and A. S. Ichimura, J. Org. Chem., 1991, 56, 3030 .

D. A. Shultz, in 'Conformational exchange modulation in trimethylenemethane-type biradicals.' ed. P. M. Lahti, New York, 1999.

P. Ferruti, D. Gill, M. P. Klein, H. H. Wang, G. Entine, and M. Calvin, J. Am. Chem. Soc., 1970, 92, 3704

C. Rancurel, H. Heise, F. H. Köhler, U. Schatzschneider, E. Rentchler, J. Vidal-Gancedo, J. Veciana, J.-P. Sutter, J. Phys. Chem., 2004, 108, 5903.

G. G. Belford, R. L. Belford, and J. F. Burkhalter, J. Magn. Reson., 1973, 11, 251.

Y. Teki, I. Fujita, T. Takui, T. Kinoshita, and K. Itoh, J. Am. Chem. Soc., 1994, 116, 11499.

L. Catala, P. Turek, J. Le Moigne, A. De Cian, and N. Kyritsakas, Tetrahedron Lett., 2000, 41, 1015.

G. M. Sheldrick, Programs for Crystal Structure Analysis, release 97-2, Institüt für Anorganische Chemie der Universität, Göttingen, Germany, 1998

L.J. Farrugia, J. Appl. Cryst., 1999, 32, 837. 
